\title{
Outcome of 11 children with ependymoblastoma treated within the prospective HIT-trials between 1991 and 2006
}

\author{
Nicolas U. Gerber • Katja von Hoff • André O. von Bueren • \\ Wiebke Treulieb - Monika Warmuth-Metz - Torsten Pietsch • \\ Niels Soerensen · Andreas Faldum • Angela Emser • Paul G. Schlegel • \\ Frank Deinlein · Rolf-Dieter Kortmann $\cdot$ Stefan Rutkowski
}

Received: 12 May 2010/Accepted: 6 August 2010/Published online: 11 February 2011

(C) Springer Science+Business Media, LLC. 2011

\begin{abstract}
Ependymoblastoma is a rare malignant brain tumor of early childhood. Data on clinical behavior and optimal treatment strategies are scarce. We report on 11 consecutively treated children with centrally confirmed diagnosis of CNS ependymoblastoma, registered between February 1994 and October 2006 to the prospective GPOHHIT multicenter brain tumor trials, and treated by multimodal regimens. Median age at diagnosis was 3.5 years (range, 1.8-5.6 years), and the median follow-up of survivors was 5.9 years (range, 2.2-12.7 years). Initial stage was M0 in 9, and M0/1 (no cerebrospinal fluid examination done) in 2 patients. Gross-total tumor resection was achieved in 7 patients, incomplete resection in 4 patients. Further primary therapy included chemotherapy in all patients, craniospinal radiotherapy in 5 patients and highdose chemotherapy in 2 patients. Tumor response to chemotherapy was observed in 1 of 4 evaluable patients. Tumor
\end{abstract}

N. U. Gerber $(\bowtie)$

Department of Oncology, University Children's Hospital,

8032 Zurich, Switzerland

e-mail: nicolas.gerber@kispi.uzh.ch

N. U. Gerber - K. von Hoff · A. O. von Bueren - W. Treulieb ·

P. G. Schlegel · F. Deinlein · S. Rutkowski

Department of Pediatrics, University of Wuerzburg,

97080 Wuerzburg, Germany

M. Warmuth-Metz

Department of Neuroradiology, University of Wuerzburg,

97080 Wuerzburg, Germany

T. Pietsch

Institute of Neuropathology, University of Bonn,

53105 Bonn, Germany progression occurred in 7 patients after a median time of 5.0 months (range, 2.5-19.2 months). Five-year progression-free survival was $36.4 \%( \pm 14.5 \%)$, 5-year overall survival $30.3 \%$ ( $\pm 15.9 \%)$. Of 4 survivors, 3 had gross-total tumor resection, and all were treated by either craniospinal radiotherapy and/or high-dose chemotherapy with autologous blood stem cell rescue. Prognosis of children with ependymoblastoma is poor, but sustained remissions have been achieved after multimodal treatment. Considerable diagnostic discrepancies between local and central pathologists underscore the importance of central review. Further studies are needed to improve survival of children with this rare malignant central nervous system tumor.

Keywords Ependymoblastoma $\cdot$ Central nervous system primitive neuroectodermal tumor $\cdot$ Children $\cdot$ Brain tumor . Cancer
N. Soerensen

Department of Pediatric Neurosurgery, University of Wuerzburg, 97080 Wuerzburg, Germany

\author{
A. Faldum · A. Emser \\ Institute for Medical Biostatistics, Epidemiology and \\ Informatics, University Medical Center, 55101 Mainz, Germany \\ R.-D. Kortmann \\ Department of Radiation Oncology, University of Leipzig, \\ 04103 Leipzig, Germany \\ K. von Hoff - A. O. von Bueren - W. Treulieb - S. Rutkowski \\ Department of Pediatric Hematology and Oncology, \\ University Medical Center Hamburg-Eppendorf, \\ 20246 Hamburg, Germany
}




\section{Introduction}

Ependymoblastoma is a rare malignant tumor of the central nervous system (CNS) described by Rubinstein in 1970 as a special type of embryonal CNS neoplasm arising in young children. So far, information on the incidence is lacking in the literature. Cytologic features of a primitive, highly cellular neuroepithelial tumor are associated with the characteristic presence of numerous ependymoblastic rosettes [1]. In the current WHO Classification of Tumours of the Central Nervous System (2007), they are classified among embryonal tumors as a subgroup of CNS primitive neuroectodermal tumors (PNET) (WHO grade IV) [2]. They occur mainly in young children; however, single cases of fetal tumors and one case in an adult have been described [3, 4]. Location is most often supratentorial, but infratentorial or spinal sites occur as well [2]. Locations outside the CNS have been reported, e.g., the cases of a congenital sacrococcygeal [5] or an ovarian tumor [6].

Ependymoblastomas show an aggressive behavior with rapid growth and craniospinal dissemination. Prognosis is generally reported as unfavorable in selected cases $[2,3,7]$. So far, clinical data on ependymoblastoma have mostly been available as case reports or have been included in analyses of other PNETs or ependymomas without separate reports on outcome. Due to the rarity of the disease, little is known about efficient treatment strategies.

Here, we report on 11 consecutive children with ependymoblastoma treated according to the prospective GPOH (German Society of Pediatric Oncology and Hematology) multicenter trials HIT'91, HIT-SKK'92, and HIT 2000 (HIT: German abbreviation for brain tumor).

\section{Patients and methods}

Patients

Eleven consecutively treated patients with ependymoblastoma diagnosed by central pathology review between February 1994 and October 2006 were treated at 11 different institutions within the GPOH multicenter brain tumor trials HIT'91 [8-10], HIT-SKK'92 [11, 12], and HIT 2000 besides patients with other malignant brain tumor histologies. There were no patients with centrally confirmed ependymoblastoma in the studies HIT-SKK' $87[9,12]$ and HIT' $88 /$ ' 89 [10, 12, 13].

\section{Surgery}

Maximal safe surgical removal of the primary tumor, depending on the anatomical location of the tumor and the condition of the child, was recommended. If intraventricular chemotherapy was part of the treatment protocol, a subcutaneous reservoir with an intraventricular catheter (an Ommaya or a Rickham reservoir) was implanted in the anterior horn of a lateral ventricle after confirmation of diagnosis. Postoperative therapy started 2-4 weeks after surgery.

\section{Diagnostic procedures}

Eighteen children with a local diagnosis of ependymoblastoma were initially registered. In 16 patients, tumor material was available for central review, the two cases without available material are not taken into account in this analysis. On central review, the diagnosis was confirmed in only 7 of those 16 cases. In the other cases, local diagnoses of ependymoblastoma were anaplastic ependymoma (5 patients), medulloblastoma (3 patients), and glioblastoma multiforme (1 patient) at central review. On the other hand, central review (conducted at the same pathology center for all patients enrolled into any of the GPOH brain tumor trials, which comprise the majority of children with any brain tumor type in Germany, Austria, and part of Switzerland) identified 4 novel cases of ependymoblastomas, which had originally been diagnosed as supratentorial CNS PNET (2 patients), medulloblastoma and glioblastoma multiforme ( 1 case each) by the local pathologist. Here, we report on all 11 patients with centrally confirmed ependymoblastoma.

According to the WHO classification, criteria for the diagnosis of ependymoblastoma was the histological finding of a primitive neuroepithelial tumor of high cellularity and the presence of multilayered rosettes composed of sheets of proliferative undifferentiated cells around a round central lumen. The tumors were characterized by immunohistochemical reactions with antibodies against GFAP, vimentin, MAP-2, synaptophysin, neurofilaments, INI-1, epithelial membrane antigen, cytokeratin, and Ki-67.

Staging included pre- and postoperative cranial magnetic resonance imaging (MRI) or computed tomography (CT), spinal MRI, and evaluation of cerebrospinal fluid (CSF) cytology as described [8-12]. Postoperative imaging no later than $48(-72) \mathrm{h}$ after surgery was recommended, as was lumbar CSF-sampling 14 days postoperatively in cases of previous positive cytology and if no negative lumbar CSF has been sampled before. The presence of microscopic CSF and macroscopic solid or laminar metastases was evaluated according to the tumor staging system of Chang and colleagues [14], with stage M0 denoting absence of metastases, stage M1 disseminated tumor cells in the CSF, stage M2 intracranial macroscopic metastases, and stage M3 spinal macroscopic metastases. Patients without macroscopic metastases who did not get CSF-cytology sampling are referred to as $\mathrm{M} 0 / 1$. 
Resection was defined as gross-total if no residual tumor was detected on postoperative imaging. Complete response was defined as the total disappearance of residual tumor during chemotherapy or at the end of treatment. Partial response was defined as a decrease of more than $50 \%$ in the tumor volume, improvement as a decrease of at least $25 \%$, but less than $50 \%$, stable disease as a decrease of less than or an increase of $25 \%$ at the most, and progressive disease as an increase of more than $25 \%$.

Besides histopathology, central review was recommended for radiology, CSF cytology, and documentation of radiotherapy and chemotherapy. During and after treatment, regular clinical and radiologic follow-up were recommended according to the respective protocols.

Treatment protocols

(1) HIT' 91: The study was open for patients between 3 and 18 years of age with malignant brain tumors newly diagnosed between August 1991 and December 1997, with a prolongation on an interim base until December 2000. Patients were randomly assigned to receive either immediate radiotherapy [35.2 Gray (Gy) on craniospinal axis/20.0 Gy boost on tumor bed] followed by 'maintenance' chemotherapy [1-(2-chloroethyl)-3cyclohexyl-1-nitrosourea (CCNU), vincristine, and cisplatin], or immediate preradiation 'sandwich' chemotherapy (ifosfamide, etoposide, methotrexate, cisplatin, and cytarabine) followed by radiotherapy, which was followed by maintenance chemotherapy in cases of residual tumor [8-10].

(2) HIT-SKK'92: Patients under 3 years of age with malignant brain tumors diagnosed between October 1992 and December 1997 received 'SKK' chemotherapy (cyclophosphamide, vincristine, methotrexate, carboplatin, and etoposide) plus intraventricular methotrexate. In cases of complete remission, no further therapy was given. Otherwise, children aged at least 18 months received radiotherapy $(35.2 \mathrm{~Gy}$ craniospinal/20.0 Gy boost); children younger than 18 months received experimental chemotherapy $[12,15]$.

(3) HIT 2000: In this prospective multicenter trial, children with ependymoblastoma were stratified together with supratentorial CNS-PNET/pineoblastoma according to age and M-stage.

\subsection{HIT 2000 below 4 years, non-metastatic disease:}

3.1.1. Patients diagnosed from January 2001 to December 2004 (before amendment) initially received 'SKK' chemotherapy. Patients with complete remission received additional cycles of 'SKK' chemotherapy without methotrexate followed by radiotherapy (24.0 Gy craniospinal/30.6 Gy boost to tumor bed plus 5.4 Gy on any residual tumor). The other patients directly received radiotherapy with concomitant vincristine, followed by maintenance chemotherapy.

3.1.2. Patients diagnosed from January 2005 onwards were treated with carboplatin, etoposide, and intraventricular methotrexate. In cases of partial or complete response, 2 high-dose chemotherapy (HDCT) courses were given (carboplatin/ etoposide and thiotepa/cyclophosphamide, plus intraventricular methotrexate), each followed by autologous blood stem cell transplantation. Subsequent radiotherapy was given to all patients who were not in complete remission after the HDCT, and optional for patients who were not in complete remission before but in complete remission after HDCT. Radiotherapy was omitted by patients already in complete remission before HDCT. Patients who did not fulfill the HDCT criteria directly received radiotherapy after the induction chemotherapy, followed by maintenance chemotherapy.

3.2 HIT 2000 older than 4 years, non-metastatic disease: Patients enrolled from January 2005 onwards were treated with 'SKK' chemotherapy, followed by hyperfractionated radiotherapy (36.0 Gy craniospinal/30 Gy boost on tumor bed plus additional boost on any residual tumor) plus concomitant weekly vincristine, followed by maintenance chemotherapy.

Informed consent was obtained from all patients and/or their legal representatives. The study protocols were approved by the Ethics Committee of the University of Wuerzburg and performed in accordance with the ethical standards laid down in the Declaration of Helsinki and its amendments.

Statistical analyses

Survival functions were estimated by the method of Kaplan and Meier. Overall survival (OS) was defined as time from primary surgery to death by any cause or last evaluation, whichever came first, and progression-free survival (PFS) as the time from primary surgery to first documented progressive disease, to death by any cause, or to last evaluation, whichever came first. Due to the small number 
of patients, no univariable or multivariable statistical analyses were done. Analysis was performed with SPSS software, version 16.0 .

\section{Results}

Patient numbers and characteristics

A total of 11 consecutively treated patients with ependymoblastoma as diagnosed by central pathology review between February 1994 and October 2006 were identified among the patients enrolled into the appropriate GPOH pediatric brain tumor studies: HIT'91, 2 patients; HITSKK'92, 1 patient; and HIT 2000, 8 patients. Details are summarized in Table 1. There were no such patients treated within the studies HIT-SKK' 87 and HIT'88/'89. Median age at diagnosis was 3.5 years (range, 1.8-5.6 years). All patients were male. Tumor location was supratentorial in 8 patients (frontal, 1; fronto-temporal, 1; fronto-parietal, 2; parietal, 3; and 3rd ventricle, 1 patient) and infratentorial in 3 patients (cerebellopontine angle, pons/cerebellar peduncle, and 4 th ventricle, 1 patient each) (Figs. 1 and 2). None of the patients had macroscopic central nervous system metastases or systemic metastases outside the central nervous system at diagnosis. Initial cerebrospinal fluid (CSF) samples were normal in 9 patients (M0 stage), and initial CSF examination was not done in 2 patients (M0/1 stage). Median follow-up time of surviving patients since diagnosis was 5.9 years (range, 2.2-12.7 years).

\section{Histopathology}

Histopathology showed the typical features of ependymoblastomas with highly cellular appearance, prominent multilayered (ependymoblastic) rosettes with central lumina. The tumors lacked ependymal features such as dominant perivascular rosettes or ependymal rosettes. Rhabdoid components or features of medulloepitheliomas such as a dominant trabecular pattern was absent. Most tumor cells expressed MAP2 and vimentin; a predominant GFAP positivity was not found. A dot- or ring-like EMA reactivity characteristic for ependymomas was not found. Cytokeratin expression was not found in a significant fraction of tumor cells. The nuclear INI-1 was retained. With antibodies against synaptophysin and neurofilamants, an abundant portion of neuropil was not demonstrated as suggested to be typical for ETANTR, a PNET variant recently described [16, 17]. KI-67 labeling demonstrated a high proliferative activity of the tumor cells, especially in the cells forming multilayered ependymoblastic rosettes.
Treatment and outcome

Tumor resection as assessed by early postoperative imaging was gross-total in 7 (all supratentorial), and incomplete in 4 patients (infratentorial, 3; supratentorial, 1 patient). Postoperative treatment according to the respective therapy protocols are described in Table 1. Postoperative first-line treatment consisted of (conventional dose) chemotherapy followed by craniospinal radiotherapy in 3 patients (nos. 1, 9 , and $10 ; 2$ of them with additional maintenance chemotherapy). Two of these patients remained in continuous complete remission during the whole therapy, one of those showing a tumor relapse a few months later. The third patient suffered a combined local and distant relapse whilst under maintenance chemotherapy after initially having shown a complete response to induction chemotherapy. Of the 2 patients (nos. 2 and 11) who were initially treated with craniospinal radiotherapy followed by chemotherapy, 1 remained in continuous complete remission, the other had additional high-dose chemotherapy followed by autologous stem cell transplantation with clinically stable disease. Thus, 3 of the 5 patients receiving craniospinal radiotherapy as part of their primary treatment remained free of tumor progression. Two patients with gross-total resection initially remained in continuous complete remission under postoperative conventional dose chemotherapy followed by high-dose chemotherapy with autologous stem cell transplantation (nos. 6 and 7), one of these patients suffered a relapse a few months later. Four patients (nos. 3, 4, 5, and 8) showed progressive disease already under primary postoperative chemotherapy. Protocol deviations can be seen from Table 1.

In summary, 7 patients had a tumor progression with a median time to progression of 5.0 months after diagnosis (range, 2.5-19.2 months), all died of disease at a median of 1.1 years after primary diagnosis (range, 0.3-3.1 years). Five-year progression-free survival probability was $36.4 \%$ $( \pm 14.5 \%)$ (Fig. 3). Five-year overall survival probability was $30.3 \%( \pm 15.9 \%)$. The pattern of relapse was combined (local and distant) in 5 cases with a spinal distant component in 3 and a supratentorial component in 2 cases; local with secondary spinal spread in 1 patient; and distant (spinal) only in 1 patient.

After tumor progression, salvage treatment consisted in partial resection followed by radiotherapy and chemotherapy in 1 patient, craniospinal radiotherapy alone in 1 patient, chemotherapy followed by craniospinal radiotherapy in 2 patients, chemotherapy, immunotherapy and hyperthermia therapy in 1 patient, chemotherapy only in 1 patient, and no salvage treatment was given in 1 case. None of the tumors responded to salvage treatment, and, accordingly, all 7 patients eventually died of tumor progression. 


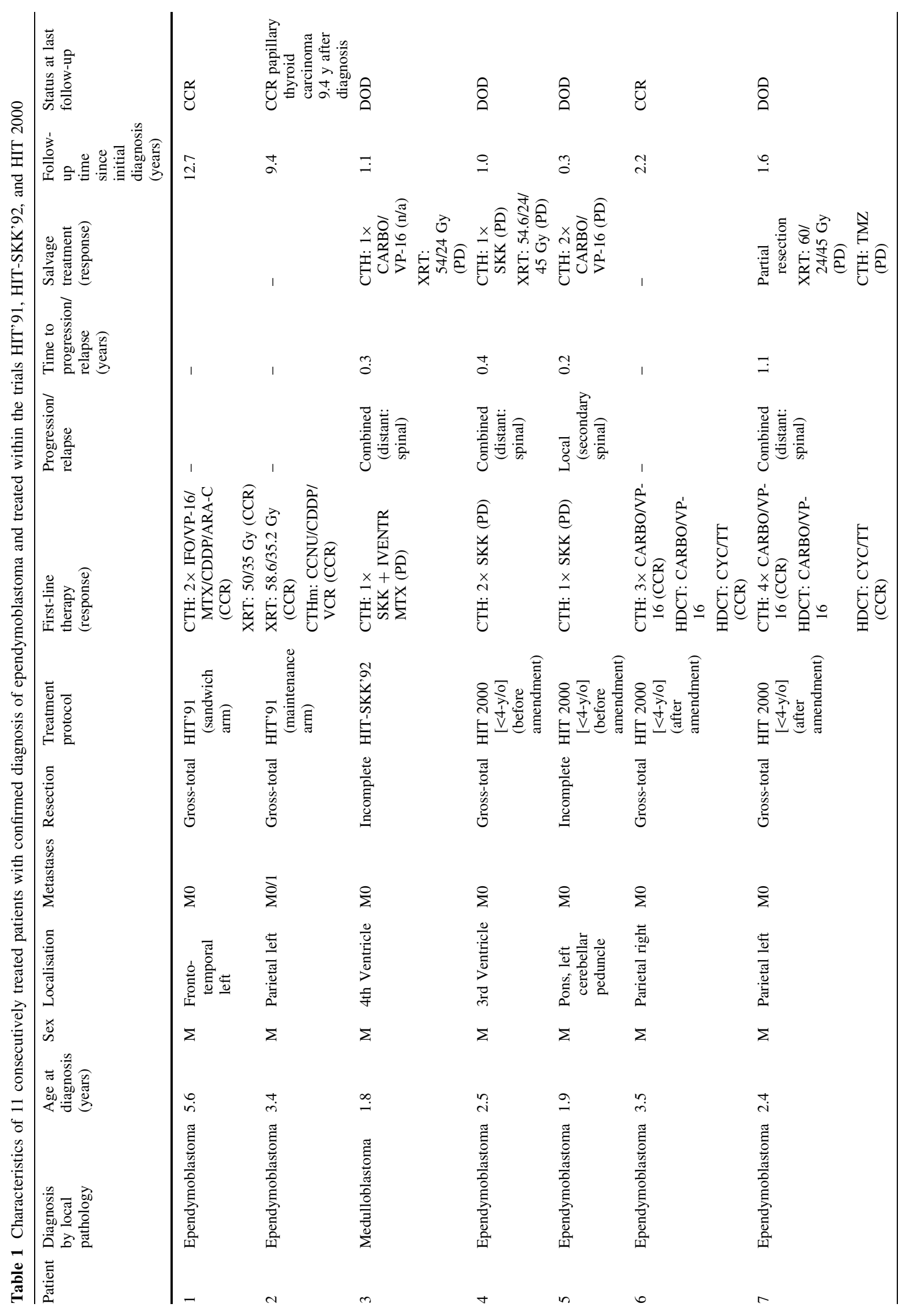




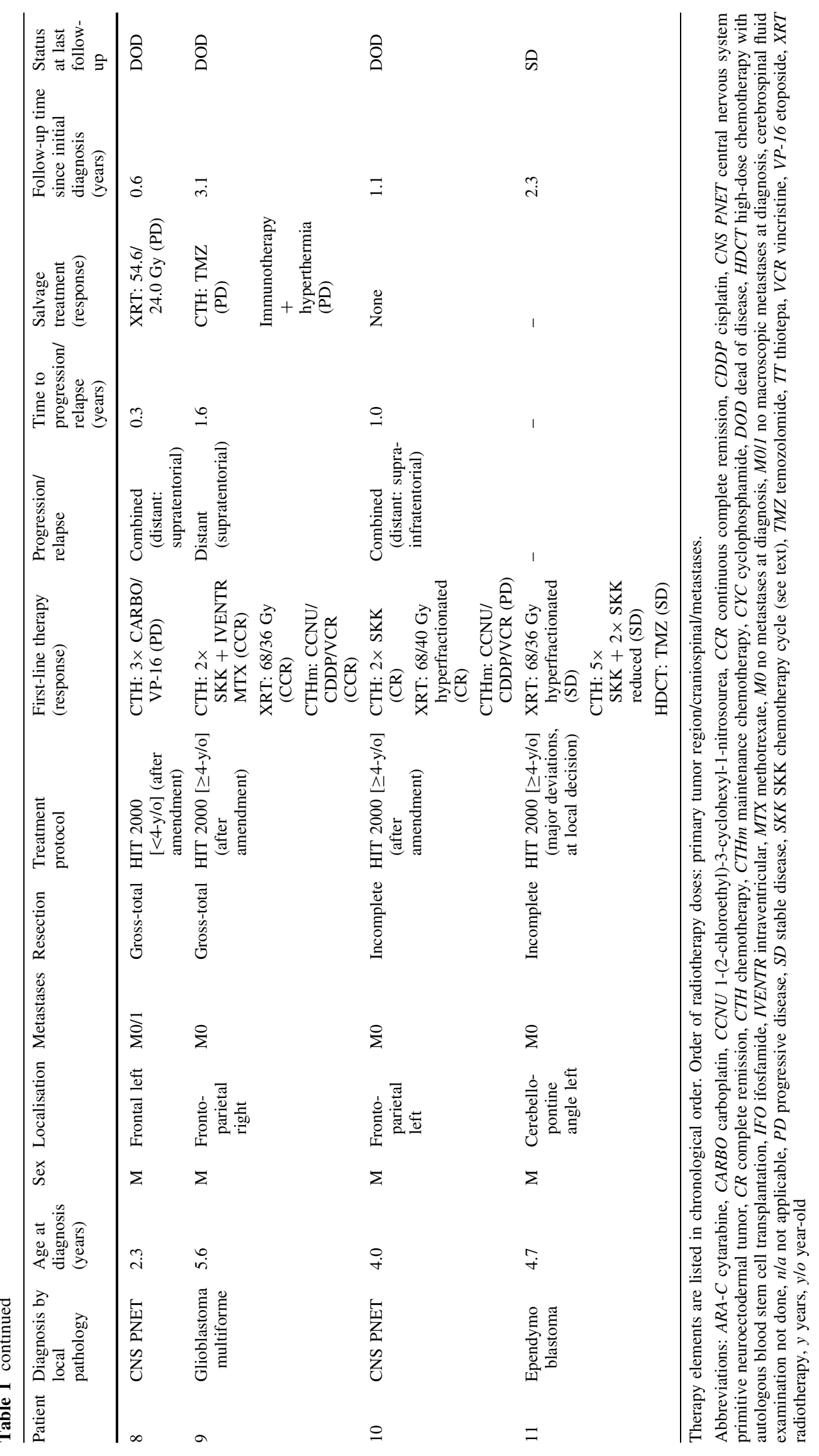


Fig. 1 a Preoperative axial T2-weighted MRI of patient no. 8 showing low signal intensity characteristic for a highly cellular tumor in the solid lateral parts of the left frontal tumor. The central high signal areas represent necrotic parts. b The medulla oblongata is displaced to the right by a probably highly cellular tumor within the left cerebellopontine cistern showing an inhomogeneous reduced signal on this preoperative axial T2-weighted MRI of patient no. 11. The basilar artery is partially encased by tumor
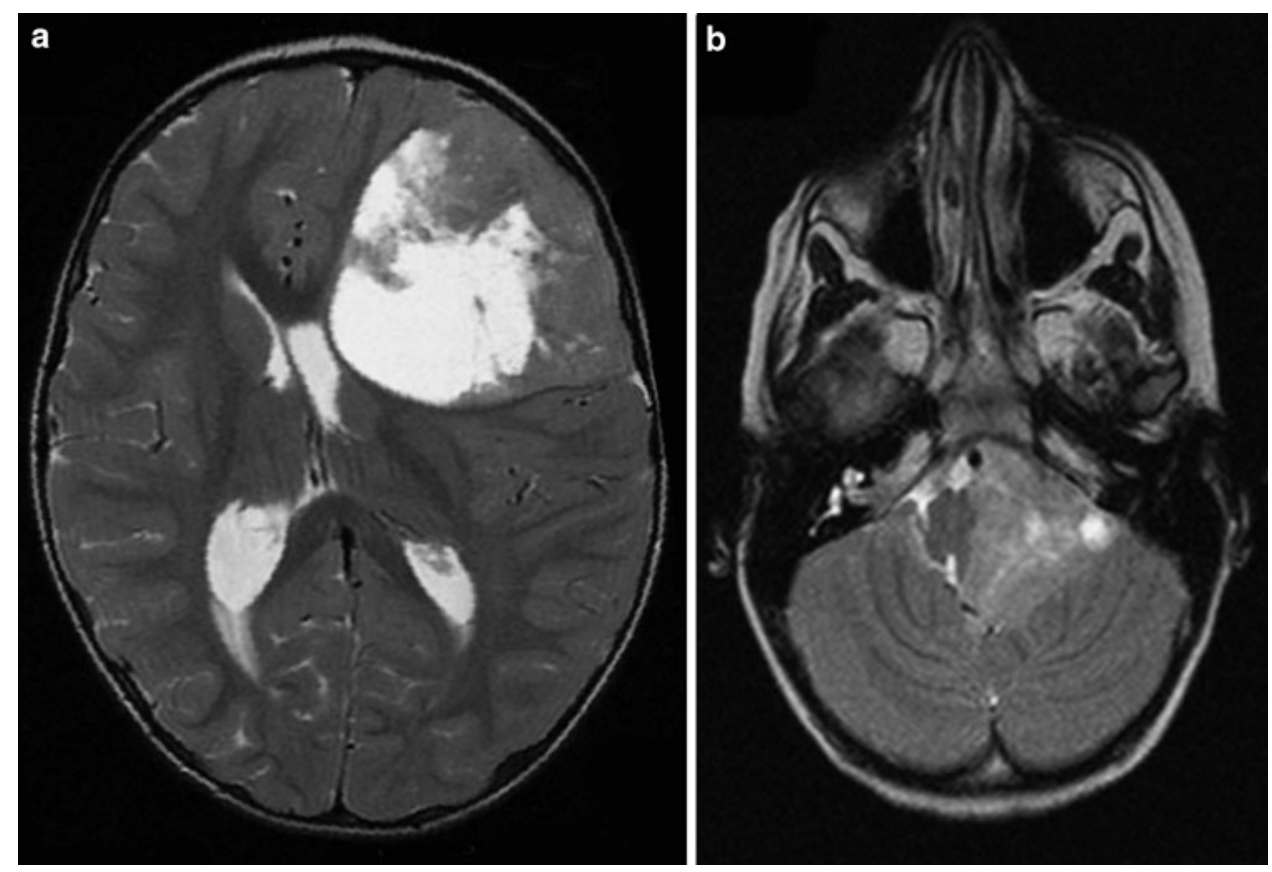

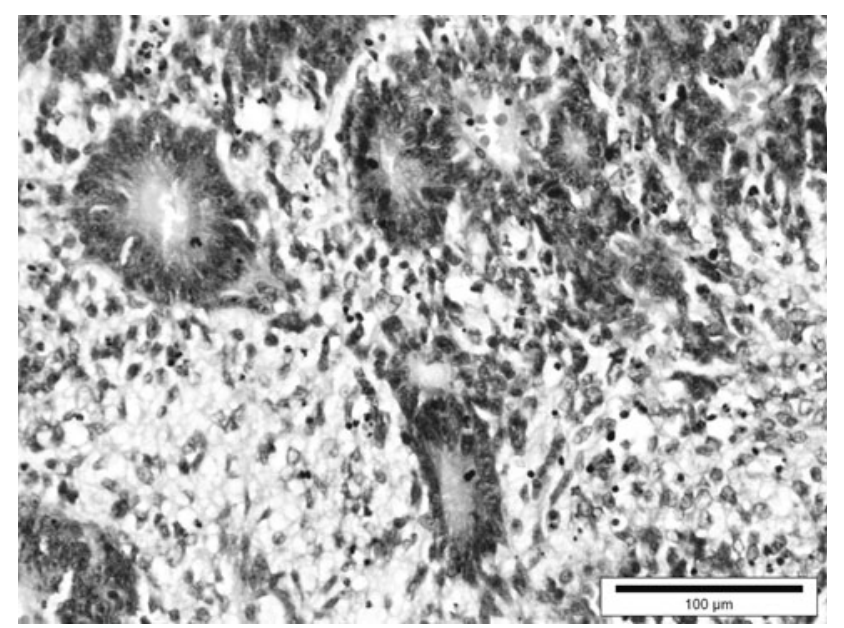

Fig. 2 Histology of case no. 10. Hematoxylin and eosin staining illustrates primitive neuroepithelial tumor composed of immature progenitor cells forming numerous multilayered proliferative rosettes with central lumina (ependymoblastomatous rosettes)

Tumor response to adjuvant treatment could be assessed in those 4 patients with incomplete tumor resection. One patient initially showed a complete response to 2 cycles of 'SKK' chemotherapy, which was maintained during radiotherapy, but he relapsed during maintenance chemotherapy. One patient had continuous stable disease under craniospinal radiotherapy, 7 cycles of 'SKK' chemotherapy, and high-dose chemotherapy with temozolomide until last follow-up 2.3 years after diagnosis. Two patients showed early progression under 'SKK' chemotherapy.

Four patients were alive at last follow-up, 3 of them in continuous complete remission $(2.2,9.4$, and 12.7 years after diagnosis) and 1 with stable disease (2.3 years after diagnosis). All the 3 patients with complete remission at last follow-up initially had gross-total tumor resection followed by intensive adjuvant treatment including either craniospinal radiotherapy (2 patients) or 2 successive highdose chemotherapy courses with autologous blood stem cell transplantation ( 1 patient). One survivor, who had been treated with craniospinal radiotherapy, was diagnosed with papillary thyroid carcinoma 9.4 years after diagnosis of the ependymoblastoma.

\section{Discussion}

Here, we report on 11 consecutively treated children with ependymoblastoma diagnosed between 1994 and 2006 and treated within the prospective multicenter GPOH HITstudies for malignant brain tumors of childhood. To our knowledge, this is the largest original series on this rare tumor entity. Our study confirms the aggressiveness of this tumor and unfavorable survival rates described in individual cases by others $[2,3,7]$. Despite intensive multimodal treatment, 7 patients died of tumor progression. Tumor response to treatment was assessable in all 4 patients with less than gross-total tumor resection. A complete response to chemotherapy was observed in 1 patient; however, this patient relapsed after further radiotherapy and chemotherapy. Two patients had an early progression during chemotherapy, while the fourth patient had stable disease under radiotherapy and chemotherapy including high-dose chemotherapy. Progressions occurred early at a median time of 

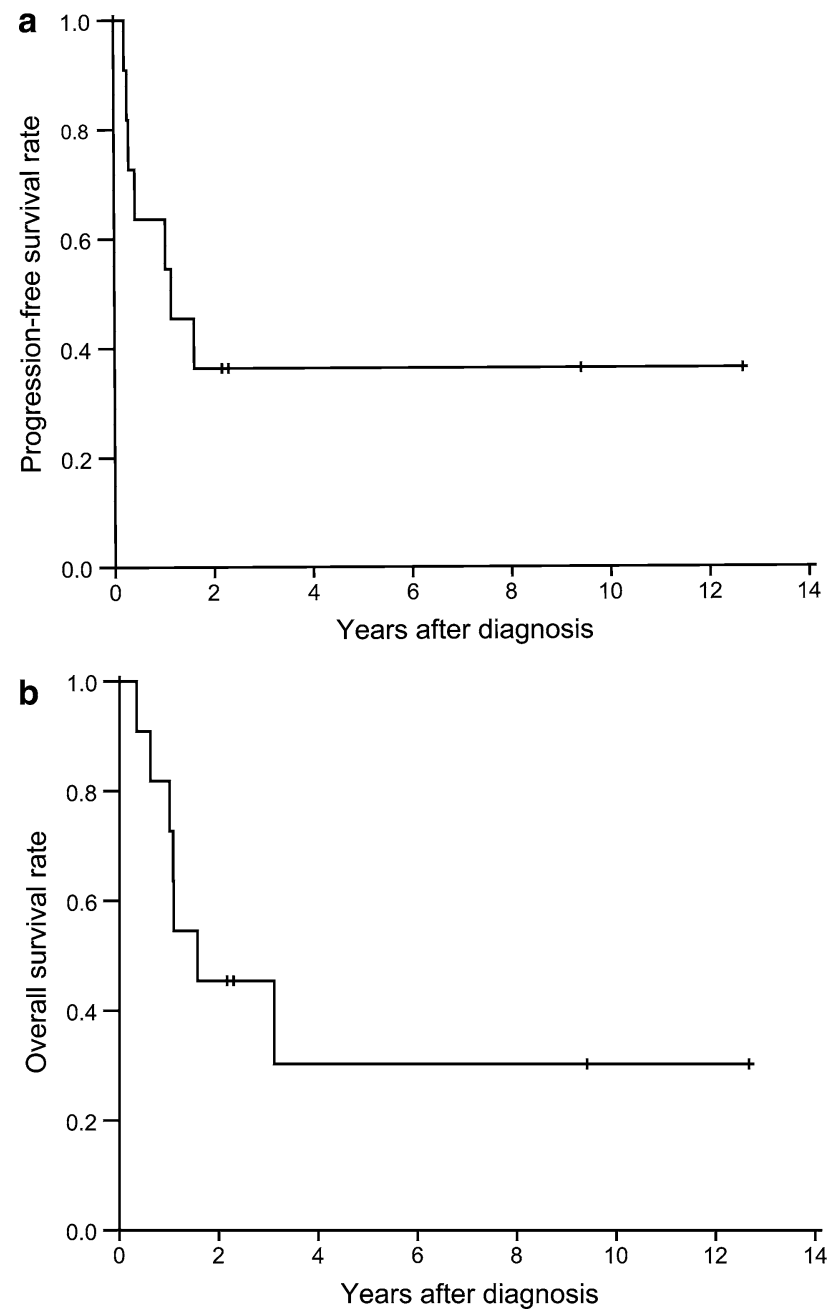

Fig. 3 Progression-free (a) and overall (b) survival rates of 11 patients with ependymoblastomas

5 months after diagnosis, most patients still being under treatment. Despite preceding systemic chemotherapy in all and craniospinal radiotherapy in 2 patients, most of the progressions had a distant component within the CNS. Salvage treatment, which was multimodal in most patients, including chemotherapy in 5 cases, and craniospinal irradiation in 4 cases, did not lead to tumor responses, and finally all patients with tumor progression died of disease.

Four patients survived at a median time of 6 years after diagnosis, of which 3 patients remain in complete remission. They all received intensive multimodal treatment consisting of surgery and chemotherapy in all cases, radiotherapy including 35-36 Gy to the craniospinal axis plus a local boost in 3 patients, and double HDCT with autologous blood stem cell transplantations in 1 patient. All but 1 surviving patients had a primarily gross-total resection, and only those 3 patients were in complete remission at last follow-up. Three out of 5 patients who received radiotherapy as part of their primary treatment are still alive, 2 in complete remission 9.4 and 12.7 years after diagnosis. In contrast, 5 patients out of 6 died after chemotherapy alone. One patient with previous radiotherapy developed a papillary thyroid carcinoma 9 years after diagnosis.

Comparison of our data with other data is only possible to a limited degree, as ependymoblastomas are very rare tumors, having been reported either as single case reports, frequently with no or only scant data on treatment and follow-up, or as part of larger reports on CNS PNET, mostly including a maximum of 1 to 3 children with ependymoblastoma, the outcome usually not being separately accounted for [7, 18-24]. The largest series dates from 1985 assembling 12 cases, which had been partially previously published by other authors in the pre-MRI era between 1970 and 1980 [3]. Age ranges were comparable to our study with predominantly young children between fetal age and 5 years old; however, with the exception of one 16-year-old and one 32-year-old patient. Seven patients were male, 5 were female. Localization was supratentorial in 8 , infratentorial in 3, and both combined in 1 case. Only 7 patients had surgery as part of the treatment regimen, 5 had radiotherapy, and 3 had chemotherapy. Median overall survival was 12 months, with longer survival of those with radiotherapy or chemotherapy. All patients died of progression except for 1 (with a short follow-up time). Interestingly, 1 patient had extracranial tumor growth at the craniotomy site and pulmonary metastases at follow-up. Our results compare favorably to this series, probably due to the more aggressive multimodal treatment regimens applied to the patients in our series. Histology of 5 patients with ependymoblastomas was described by Cruz-Sanchez in 1988 [25]. Apart from age at diagnosis (0.7-14 years), sex (3, male; 2, female), and tumor localization (4, supratentorial; 1 infratentorial; all related to the ventricles), no clinical data are given. As in our series, localization of ependymoblastomas was more often supratentorial than infratentorial [3, 7, 25, 26]; however, single cases of transtentorial extension, spinal localization, and primary leptomeningeal spread have been described [3, 27, 28]. Primary extra-CNS localization has been observed, including ovary and sacrococcygeal soft tissue [5, 29, 30]. Metastatic spread occurs mostly in the CNS; however, single cases of pulmonary and lymph node metastases have also been found [3, 26, 31, 32]. In many case reports, no information is given on treatment and follow-up. In most of those cases where such information is available, the patients died within several months to a few years. Due to the small total of patients, heterogeneous patient and treatment characteristics as well as availability of information, no prognostic factors can be detected.

The diagnoses of all patients reported in this study have been made by central reference pathology according to the 
current WHO classification of brain tumors [2]. There were considerable discrepancies between diagnoses from local and reference pathologists. In 9 of 16 patients with local diagnosis of ependymoblastoma and tumor material available for central review, the diagnoses of anaplastic ependymoma, medulloblastoma, and glioblastoma were made by central review. Thus, only 7 out of $16(44 \%)$ of local diagnoses of ependymoblastoma could be centrally confirmed. On the other hand, the central pathologist identified 4 ependymoblastoma patients with former local diagnoses of CNS PNET, medulloblastoma, and glioblastoma multiforme. Similar diagnostic challenges have been observed by others: in a trial enrolling 32 patients with centrally diagnosed ependymoma, 9 of them were initially diagnosed as ependymoblastoma by the local pathologists [33], and other divergent diagnoses included medulloepithelioma [18]. These discrepancies strongly support the importance of central pathology review, not only in the context of clinical trials. The importance of a precise histological diagnosis is additionally underscored by the fact that there are no known clinical or radiological features distinguishing ependymoblastomas from other tumor types. It is essential that publications about ependymoblastoma must be studied carefully, as some case reports might in fact represent other tumor entities due to incorrect diagnosis. Furthermore, ependymoblastomas have wrongly been defined-not in accordance with the current WHO Classification of Tumours of the Central Nervous System-as high-grade ependymomas, as done in a publication describing the largest series of patients with "ependymoblastoma" [34].

The tumors described here fulfill the histological criteria for the diagnosis of ependymoblastoma as defined by the current WHO Classification of Brain Tumors [2]. Recently, a primitive tumor characterized as embryonal tumors with abundant neuropil and true rosettes (ETANTR) has been described [17]. Some authors believe that they have identified a novel histologic entity with combined features of ependymoblastoma and neuroblastoma, heavily cellular regions and fibrillar neuropil-like areas containing mitotically active and multilayered ependymoblastomatous rosettes (here called "true" rosettes), and with poor clinical outcome [16, 17, 35, 36]. However, this phenotype largely overlaps with original histological descriptions of such tumors by Rubinstein in 1970 [1] and may represent the identical tumor entity. Therefore, the ETANTR has not been accepted as a novel entity by the current WHO Classification of Tumours of the Central Nervous System [2, 16, 17, 35, 36].

Our study has several strengths: to the best of our knowledge, it is the largest series of recently published patients, histologies were centrally reviewed, all patients diagnosed with ependymoblastoma within the indicated time period are reported on, treatments were given according to contemporary protocols for PNET, and follow-up time of survivors is relatively long. However, we are also aware of limitations: the number of patients is small, hence possible prognostic factors cannot be analyzed statistically. Due to the time period covered by patients from our series, patients were not treated homogeneously.

Nevertheless, we believe that our study contributes to the understanding of this rare disease, and it demonstrates that the disease may be cured in some of the patients. Local tumor control seems to be of importance in this disease, as most survivors had gross-total tumor resection. Furthermore, all survivors had aggressive postoperative treatment containing radiotherapy and/or high-dose chemotherapy with autologous blood stem cell rescue. Radiotherapy as part of primary treatment seemed to have a positive effect on survival; however, it did not confer a sustained benefit as part of salvage strategies in patients with tumor progression during initial postoperative chemotherapy. A future approach using upfront radiation therapy seems to be a promising strategy to improve outcome. The selection of target volumes and necessary dose prescriptions are still open questions. For instance, restrictions of the irradiation field to the tumor bed only, which have been successfully established in treatment concepts for children with intracranial ependymoma with clinically relevant benefits in long-term toxicities and neuropsychological deficits, might be an option worth investigating, especially in very young children [37, 38]. Also, the role of chemotherapy has to be further clarified: whereas one patient survived after highdose chemotherapy without radiotherapy and response to chemotherapy was observed in another patient, 4 early relapses were observed during postoperative chemotherapy in our series. It thus seems that, at least in patients with incomplete tumor resection, any pre-irradiation induction chemotherapy should be kept short, as the risk of early relapse during chemotherapy may be considerable.

In summary, our series show that some patients with ependymoblastomas may be cured by surgical treatment followed by either a combination of radiotherapy and chemotherapy or by chemotherapy including high-dose therapy without irradiation. However, it remains unclear which of these approaches is the most promising, and the optimal sequence of the different therapy modalities has yet to be defined. Clearly, further research on clinical behavior, tumor biology and potential prognostic factors is warranted, and effective risk-adapted treatment recommendations, optimizing the balance between treatment intensity and adverse treatment effects, need to be improved.

\section{References}

1. Rubinstein LJ (1970) The definition of the ependymoblastoma. Arch Pathol 90:35-45 
2. Louis DN, Ohgaki H, Wiestler O, Cavenee WKE (2007) WHO Classification of Tumours of the Central Nervous System. IARC Press, Lyon

3. Mork SJ, Rubinstein LJ (1985) Ependymoblastoma. A reappraisal of a rare embryonal tumor. Cancer 55:1536-1542

4. Isaacs H (2009) Fetal brain tumors: a review of 154 cases. Am J Perinatol 26:453-466

5. Santi M, Bulas D, Fasano R, Ponsky T, Sandler A, Richter E, Rushing EJ (2008) Congenital ependymoblastoma arising in the sacrococcygeal soft tissue: a case study. Clin Neuropathol 27:78-82

6. Morovic A, Damjanov I (2008) Neuroectodermal ovarian tumors: a brief overview. Histol Histopathol 23:765-771

7. Cervoni L, Celli P, Trillo G, Caruso R (1995) Ependymoblastoma: a clinical review. Neurosurg Rev 18:189-192

8. Kortmann RD, Kuhl J, Timmermann B, Mittler U, Urban C, Budach V, Richter E, Willich N, Flentje M, Berthold F, Slave I, Wolff J, Meisner C, Wiestler O, Sorensen N, Warmuth-Metz M, Bamberg M (2000) Postoperative neoadjuvant chemotherapy before radiotherapy as compared to immediate radiotherapy followed by maintenance chemotherapy in the treatment of medulloblastoma in childhood: results of the German prospective randomized trial HIT'91. Int J Radiat Oncol Biol Phys 46:269-279

9. Rutkowski S, Gerber NU, von Hoff K, Gnekow A, Bode U, Graf N, Berthold F, Henze G, Wolff JE, Warmuth-Metz M, Soerensen N, Emser A, Ottensmeier H, Deinlein F, Schlegel PG, Kortmann RD, Pietsch T, Kuehl J (2009) Treatment of early childhood medulloblastoma by postoperative chemotherapy and deferred radiotherapy. Neurooncology 11:201-210

10. Timmermann B, Kortmann RD, Kuhl J, Meisner C, Dieckmann K, Pietsch T, Bamberg M (2002) Role of radiotherapy in the treatment of supratentorial primitive neuroectodermal tumors in childhood: results of the prospective German brain tumor trials HIT 88/89 and 91. J Clin Oncol 20:842-849

11. Hinkes BG, von Hoff K, Deinlein F, Warmuth-Metz M, Soerensen N, Timmermann B, Mittler U, Urban C, Bode U, Pietsch T, Schlegel PG, Kortmann RD, Kuehl J, Rutkowski S (2007) Childhood pineoblastoma: experiences from the prospective multicenter trials HIT-SKK87, HIT-SKK92 and HIT91. J Neurooncol 81:217-223

12. Timmermann B, Kortmann RD, Kuhl J, Rutkowski S, Meisner C, Pietsch T, Deinlein F, Urban C, Warmuth-Metz M, Bamberg M (2006) Role of radiotherapy in supratentorial primitive neuroectodermal tumor in young children: results of the German HIT-SKK87 and HIT-SKK92 trials. J Clin Oncol 24: $1554-1560$

13. Kuhl J, Muller HL, Berthold F, Kortmann RD, Deinlein F, Maass E, Graf N, Gnekow A, Scheurlen W, Gobel U, Wolff JE, Bamberg M, Kaatsch P, Kleihues P, Rating D, Sorensen N, Wiestler OD (1998) Preradiation chemotherapy of children and young adults with malignant brain tumors: results of the German pilot trial HIT'88/'89. Klin Padiatr 210:227-233

14. Chang CH, Housepian EM, Herbert C Jr (1969) An operative staging system and a megavoltage radiotherapeutic technic for cerebellar medulloblastomas. Radiology 93:1351-1359

15. Rutkowski S, Bode U, Deinlein F, Ottensmeier H, WarmuthMetz M, Soerensen N, Graf N, Emser A, Pietsch T, Wolff JE, Kortmann RD, Kuehl J (2005) Treatment of early childhood medulloblastoma by postoperative chemotherapy alone. N Engl J Med 352:978-986

16. Gessi M, Giangaspero F, Lauriola L, Gardiman M, Scheithauer BW, Halliday W, Hawkins C, Rosenblum MK, Burger PC, Eberhart CG (2009) Embryonal tumors with abundant neuropil and true rosettes: a distinctive CNS primitive neuroectodermal tumor. Am J Surg Pathol 33:211-217
17. Eberhart CG, Brat DJ, Cohen KJ, Burger PC (2000) Pediatric neuroblastic brain tumors containing abundant neuropil and true rosettes. Pediatr Dev Pathol 3:346-352

18. Marec-Berard P, Jouvet A, Thiesse P, Kalifa C, Doz F, Frappaz D (2002) Supratentorial embryonal tumors in children under 5 years of age: an SFOP study of treatment with postoperative chemotherapy alone. Med Pediatr Oncol 38:83-90

19. Cruz-Sanchez FF, Rossi ML, Hughes JT, Moss TH (1991) Differentiation in embryonal neuroepithelial tumors of the central nervous system. Cancer 67:965-976

20. Dai AI, Backstrom JW, Burger PC, Duffner PK (2003) Supratentorial primitive neuroectodermal tumors of infancy: clinical and radiologic findings. Pediatr Neurol 29:430-434

21. Johnston DL, Keene DL, Lafay-Cousin L, Steinbok P, Sung L, Carret AS, Crooks B, Strother D, Wilson B, Odame I, Eisenstat DD, Mpofu C, Zelcer S, Huang A, Bouffet E (2008) Supratentorial primitive neuroectodermal tumors: a Canadian pediatric brain tumor consortium report. J Neurooncol 86:101-108

22. Liu HM, Boogs J, Kidd J (1976) Ependymomas of childhood. I. Histological survey and clinicopathological correlation. Childs Brain 2:92-110

23. Mikaeloff Y, Raquin MA, Lellouch-Tubiana A, Terrier-Lacombe MJ, Zerah M, Bulteau C, Habrand JL, Kalifa C (1998) Primitive cerebral neuroectodermal tumors excluding medulloblastomas: a retrospective study of 30 cases. Pediatr Neurosurg 29:170-177

24. Varan A, Akalan N, Soylemezoglu F, Zorlu F, Yalcin B, Akyuz C, Kutluk T, Buyukpamukcu M (2006) Central nervous system tumors in patients under three years of age: treatment results of a single institute. Pediatr Neurosurg 42:89-94

25. Cruz-Sanchez FF, Haustein J, Rossi ML, Cervos-Navarro J, Hughes JT (1988) Ependymoblastoma: a histological, immunohistological and ultrastructural study of five cases. Histopathology 12:17-27

26. Dorsay TA, Rovira MJ, Ho VB, Kelley J (1995) Ependymoblastoma. MR presentation. A case report and review of the literature. Pediatr Radiol 25:433-435

27. Wada C, Kurata A, Hirose R, Tazaki Y, Kan S, Ishihara Y, Kameya T (1986) Primary leptomeningeal ependymoblastoma. Case report. J Neurosurg 64:968-973

28. Monteferrante ML, Shimkin PM, Fichtenbaum C, Kleinman GM, Lipow KI (1991) Tentorial traversal by ependymoblastoma. Am J Neuroradiol 12:181

29. Murphy MN, Dhalla SS, Diocee M, Halliday W, Wiseman NE, deSa DJ (1987) Congenital ependymoblastoma presenting as a sacrococcygeal mass in a newborn: an immunohistochemical, light and electron microscopic study. Clin Neuropathol 6:169-173

30. Kleinman GM, Young RH, Scully RE (1993) Primary neuroectodermal tumors of the ovary. A report of 25 cases. Am J Surg Pathol 17:764-778

31. Shyn PB, Campbell GA, Guinto FC Jr, Crofford MJ (1986) Primary intracranial ependymoblastoma presenting as spinal cord compression due to metastasis. Childs Nerv Syst 2:323-325

32. Campbell AN, Chan HS, Becker LE, Daneman A, Park TS, Hoffman HJ (1984) Extracranial metastases in childhood primary intracranial tumors. A report of 21 cases and review of the literature. Cancer 53:974-981

33. Robertson PL, Zeltzer PM, Boyett JM, Rorke LB, Allen JC, Geyer JR, Stanley P, Li H, Albright AL, McGuire-Cullen P, Finlay JL, Stevens KR Jr, Milstein JM, Packer RJ, Wisoff J (1998) Survival and prognostic factors following radiation therapy and chemotherapy for ependymomas in children: a report of the Children's Cancer Group. J Neurosurg 88:695-703

34. Dohrmann GJ, Farwell JR, Flannery JT (1976) Ependymomas and ependymoblastomas in children. J Neurosurg 45:273-283

35. Judkins AR, Ellison DW (2010) Ependymoblastoma: dear, damned, distracting diagnosis, farewell! Brain Pathol 20:133-139 
36. Cenacchi G, Giangaspero F (2004) Emerging tumor entities and variants of CNS neoplasms. J Neuropathol Exp Neurol 63:185-192

37. Merchant TE, Mulhern RK, Krasin MJ, Kun LE, Williams T, Li C, Xiong X, Khan RB, Lustig RH, Boop FA, Sanford RA (2004) Preliminary results from a phase II trial of conformal radiation therapy and evaluation of radiation-related CNS effects for pediatric patients with localized ependymoma. J Clin Oncol 22:3156-3162

38. Conklin HM, Li C, Xiong X, Ogg RJ, Merchant TE (2008) Predicting change in academic abilities after conformal radiation therapy for localized ependymoma. J Clin Oncol 26:3965-3970 